DAS LIED IM DEUTSCHEN SÜDWESTEN IM 18. JAHRHUNDERT UND IN DER ERSTEN HÄLFTE DES 19. JAHRHUNDERTS 


\section{DAS LIED IM \\ DEUTSCHEN SÜDWESTEN \\ IM 18. JAHRHUNDERT \\ UND IN DER ERSTEN HÄLFTE \\ DES 19. JAHRHUNDERTS}

Sonderdruck aus

Musik in Baden-Württemberg

Jahrbuch 2002 / Band 9

\section{Im Auftrag \\ der}

Gesellschaft für Musikgeschichte in Baden-Württemberg herausgegeben von

Georg Günther und Reiner Nägele

VERLAG J. B. METZLER STUTTGART $\cdot$ WEIMAR 


\section{GESELLSCHAFT FUR MUSIKGESCHICHTE IN BADEN-WURTTEMBERG E.V.}

Schulberg 2, 72070 Túbingen

Prăsident: Hermann Fünfgeld

Vorsitzender des Wissenschaftlichen Beirats: Prof. Dr. Manfred Hermann Schmid

ISBN 978-3-476-98866-9

ISBN 978-3-476-98865-2 (eBook)

DOI 10.1007/978-3-476-98865-2

Sonderdruck des Jahrbuchs »Musik in Baden-Württemberg « (2002), im Buchhandel nicht erhaltlich

Endredaktion: Georg Gunther

Dieses Werk einschließlich aller seiner Teile ist urheberrechtlich geschutzt. Jede Verwertung außerhalb der engen Grenzen des Urheberrechtsgesetzes ist ohne Zustimmung des Verlages unzulăssig und strafbar. Das gilt insbesondere für Vervielfaltigungen, Übersetzungen, Mikroverfilmungen und die Einspeicherung und Verarbeitung in elektronischen Systemen.

Für den Inhalt der einzelnen Beitrăge einschließlich Abbildungen sind die Verfasser verantwortlich.

(C) 2002 Springer-Verlag GmbH Deutschland

Ursprünglich erschienen bei J. B. Metzlersche Verlagsbuchhandlung und Carl Ernst Poeschel Verlag GmbH 2002

www.metzlerverlag.de info@metzlerverlag.de

Einbandgestaltung: Willy Loffelhardt

Satz: Georg Günther

J. B. Metzler Stuttgart · Weimar 


\section{INHALT}

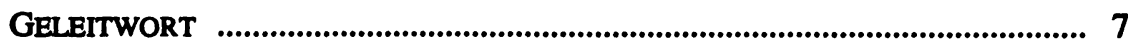

„FRUHLING LÄSST SEIN BLAUES BAND ...*

Das Lied im deutschen Südwesten

\section{HARTMUT SCHICK}

»Mehr Naturschrey als Kunst« - Zum Liedschaffen von Christian

Friedrich Daniel Schubart

WALTHER DÜR

Johann Rudolf Zumsteeg (1760-1802) oder: Besonderheiten des

schwäbischen Liedes im Ausgang des 18. Jahrhundets

FRIRDHELM BRUSNIAK

Zwischen höfischer und bürgerlicher Musikkultur - Zu Johann Amons »Wallersteiner Jägerliedern« Opus 103

REINER NÄGELE

Das Populäre und das Klassische - oder Antwort auf die Frage:

Ob Kreutzer ein besserer Komponist als Schubert sei?

ANDREAS TRAUB

Vom Misslingen

MARTINA REBMANN

\#Ihr Kompositionstalent hat kraftvolle Probe geliefertк - Zum Lied-

schaffen der Stuttgarter Komponistin Emilie Zumsteeg (1796-1857) ..... 83

MIRJAM SPRINGER

»Klang-Farben - Lyrik und Lieder von Annette von

Droste-Hülshoff (1797-1848)

HARALD PFEIFFER

Der Liederkomponist Louis Hetsch (1806-1872) - Beispiel eines süddeutschen Kleinmeisters im 19. Jahrhundert

HARALD KREBS

"Meine Lieder sind mein Tagebuch « - Autobiographisches in den Liedern und Liedmanuskripten Josephine Langs

MANFRED HERMANN SCHMID

Die Instrumente der Liedbegleitung

THOMAS KABISCH

Verzweigungen und Scharniere - Beethoven liest und komponiert Goethe

GREGOR WITTKOP

»Mignons Lied火 vor dem Hintergrund der Einheitsproblematik in

*Wilhelm Meisters Lehrjahre« 
Die Autoren der Beitrăge

GESELLSCHAFT FÜR MUSIKGESCHICHTE IN BADEN-WÜRTTEMBERG E.V.

Vorstand und Beirat 180

\section{REGISTER}

1. Personen ................................................................................................ 181

2. Orte …................................................................................................... 184 


\section{GELEITWORT}

Die Gesellschaft für Musikgeschichte in Baden-Württemberg verstärkt ihre Aufgaben insoweit, als sie zusătzlich zu den bisherigen Publikationen Vortragsveranstaltungen und Symposien veranstaltet zu bestimmten Sachthemen. So wurde in der Zeit vom 24. bis 27. Mai 2001 im Kloster Schöntal eine Arbeitstagung mit dem Thema DDas Lied im deutschen Sudwesten im 18. Jahrhundert und in der ersten Halfte des 19. Jahrhundertsk durchgeführt. Dank einer vorzulglichen Zusammenarbeit mit dem Verein für Württembergisch Franken und dem Bildungshaus des Klosters Schöntal konnte diese Veranstaltung als Erfolg verstanden werden.

Die bei dieser Tagung gehaltenen Referate sind im Jahrbuch Musik in Baden-Württemberg 2002 - Band $9 \ll$ wiedergegeben. Es besteht nun der Wunsch und das Bedürfnis, diesen speziellen Teil des Jahrbuches den Veranstaltern und einem daruber hinaus interessierten Kreis zur Verfügung zu stellen.

Dieser Sonderdruck wird ergänzt durch eine vom Südwestrundfunk, der Gesellschaft für Musikgeschichte in Baden-Württemberg und Cavalli Records herausgebrachte CD mit Liedern aus dem deutschen Südwesten, die bei einem während der Tagung veranstalteten Konzert vorgetragen wurden.

Mein besonderer Dank gilt den Herren Georg Güther M.A. und Dr. Reiner Nägele für die gewohnt zuverlässige und umsichtige Schriftleitung des Jahrbuchs 2002 und dieses Sonderdrucks. Ebenso danke ich Herrn Prof. Ulf Scharlau und der beteiligten Redaktion des SWR für deren Bemühungen um das $\mathrm{Zu}$ standekommen der CD.

Hermann Fünfgeld

Prösident der Gesellschaft für Musikgeschichte in Baden-Württemberg 